\title{
OPTIMAL CONTROL FOR VEHICLE CRUISE SPEED TRANSFER
}

\author{
Tiago R. Jorge \\ INESC-ID \\ R. Alves Redol 9 \\ 1000-029 Lisboa, Portugal \\ email: tiago.nuno.jorge@ist.utl.pt
}

\author{
João M. Lemos \\ INESC-ID/IST \\ R. Alves Redol 9 \\ 1000-029 Lisboa, Portugal \\ email: jlml@inesc.pt
}

\author{
Miguel Barão \\ INESC-ID/Univ. vora \\ R. Alves Redol 9 \\ 1000-029 Lisboa, Portugal \\ email: mjsb@ramses.inesc.pt
}

\begin{abstract}
The contribution of this paper consists in a procedure to solve the optimal cruise control problem that consists in transferring the car velocity between two specified values, in a fixed interval of time, with minimum fuelconsumption. The solution is obtained by applying a recursive numerical algorithm that provides an approximation to the condition provided by Pontryagin's Optimum Principle. This solution is compared with the one obtained by using a reduced complexity linear model for the car dynamics that allows an exact ("analytical") solution of the corresponding optimal control problem. This work has been performed within the framework of activity 2.4.1 - Smart drive control of project SE2A - Nanoelectronics for Safe, Fuel Efficient and Environment Friendly Automotive Solutions, ENIAC initiative.
\end{abstract}

\section{KEY WORDS}

Optimization, Nonlinear Control, Automotive, Cruise Control.

\section{Introduction}

Growing concerns with environment protection and energy optimization, together with recent progress in automotive technology (including electronics, sensors, actuators and fault tolerant software) is boosting research on control for automotive applications. Along this line, recent papers address various aspects of cruise control based on Predictive and Optimal Control [1, 2, 3, 4, 5, 6].

The contribution of this paper consists in a procedure to solve the optimal cruise control problem of transferring the car velocity between two specified values, in a fixed interval of time, while minimizing a function of fuelconsumption.

The solution is obtained by applying a recursive numerical algorithm that provides an approximation to the necessary conditions of Pontryagin's Optimum Principle.

This solution is compared with the one obtained in [7] by using a reduced complexity linear model for the car dynamics that allows an exact ("analytical") solution of the corresponding optimal control problem.

The problem solved assumes that a constant gear is applied during the whole time interval considered. This is a step of the solution of the more general dynamic opti- mization problem in which the time at which gears switch is also a variable to be optimized. In that case the problem becomes of hybrid optimization type.

In addition to being one of the steps in the solution of the more general problem, the results in this paper concerning the use of linearized simplified models have the interest of characterizing the error obtained with a method that is significantly faster than the nonlinear one.

The remainder of this paper is organized as follows. Section 2.1 presents the nonlinear car model used in the simulations, together with a procedure to obtain a reduced complexity linearized model. Section 3 presents the numerical optimization algorithm used to solve the optimal control problem, for any nonlinear or linear system. Section 4 describes the minimum fuel velocity transfer problem and compares the optimal control signals obtained by optimizing the original nonlinear model and the reduced complexity linear model. Finally, section 5 draws some conclusions on the obtained results and the use of the optimization algorithm for nonlinear models.

\section{Car models}

Two models are considered for the vehicle dynamics: A one-dimensional car nonlinear model and its linearized version. The nonlinear model is fully described in [7].

\subsection{One-dimensional nonlinear car model}

This section describes a one-dimensional model for a diesel car, with the following inputs:

- fuel flow as controlled input $[L / s]$;

- selected gear (manual gearbox is assumed);

- terrain inclination $[\mathrm{rad}]$ and wind speed $[\mathrm{m} / \mathrm{s}]$ as disturbances.

The main output of the model is the car speed. Other quantities available from this model are:

1. engine rotational speed $[\mathrm{rad} / \mathrm{s}]$;

2. engine torque $[\mathrm{Nm}]$;

3. engine power $[k W]$; 


\section{4. fuel consumption $[L / 100 \mathrm{~km}]$.}

The dynamic model is build from elementary physical principles using information publicly available for a Toyota Avensis 2.0 D-4D SW for a 2007 model. All physical quantities are measured in SI units. A table containing the values used for the constant parameters of the model is given at the end of this section.

The evolution of the car speed depends of the forces applied. The forces considered are: traction force $F(t)$, gravitational force and aerodynamic drag $F_{a}(t)$.

$$
\dot{v}(t)=-9.8 \sin (\theta)+\frac{1}{m}\left(F(t)-F_{a}(t)\right)
$$

where $\theta$ is the terrain inclination. Aerodynamic drag is assumed to be given by

$$
F_{a}(t)=\frac{1}{2} \rho A C_{d}\left(v(t)-v_{\text {wind }}(t)\right)^{2},
$$

where $\rho$ is the air density, $A$ is the frontal area of the vehicle, $C_{d}$ is the drag coefficient, and $m$ is the car mass. The relation of the traction force $F(t)$ with the fuel flow $u$ is explained in appendix A for the sake of completeness. The reader is referred to [7] for the values of the parameters used.

In order to design the controller it is convenient to write model (1) in the standard non-linear state-apace form

$$
\dot{x}=f(x, u)
$$

where $x=v$ (vehicle speed) is the state, $u$ (fuel flow) is the manipulated variable and $f$ is a function defined by (1).

\subsection{Linearized car model}

Consider the following linear model for the car velocity increments around an equilibrium:

$$
\dot{\Delta v}=-a \Delta v+b \Delta u
$$

where $\Delta v$ is the incremental car speed measured in $[\mathrm{m} / \mathrm{s}]$ and $\Delta u$ the incremental fuel flow measured in $[L / s]$. Linearization is done around a working point $\left(v_{0}, u_{0}\right)$ of the nonlinear model, where $v_{0}$ is the starting vehicle velocity, at time $t=0$, for the velocity transfer problem, and $u_{0}$ is the fuel flow value that allows the car to maintain a stationary velocity $v_{0}$. Figure 1 shows the values of $u_{0}$ for a wide range of stationary velocities, for all the 6 gears. To avoid hybrid dynamics, the actual gear value used is previously selected and held constant throughout the integration of the nonlinear system state equation.

Parameters $a$ and $b$ are chosen to best approximate the nonlinear model response to a step of size $\delta u>0(\delta u$ small), around the working point $\left(v_{0}, u_{0}\right)$, i.e. using a fuel flow signal

$$
u(t)= \begin{cases}u_{0} & t<t_{s} \\ u_{0}+\delta u & t>t_{s}\end{cases}
$$

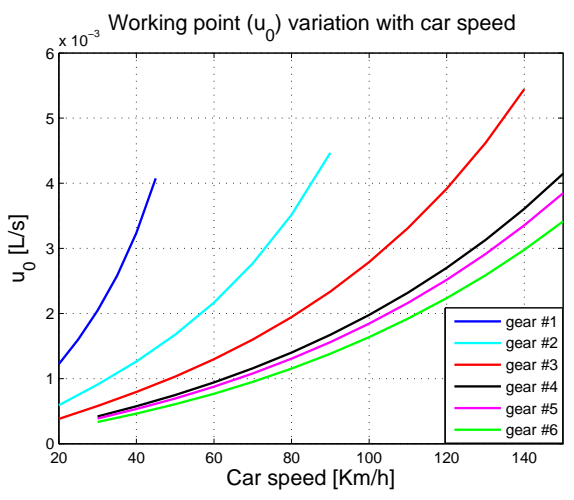

Figure 1. Working point fuel flow $u_{0}$ values for all 6 gears of the nonlinear car model.

where $t_{s}$ is the time instant when the step is applied.

The vehicle velocity evolution resembles the response of a first-order model and, given enough time, it reaches a stationary value after having increased a total of $\delta v$. The static gain of the linearized model is thus

$$
K=\frac{\delta v}{\delta u} .
$$

The response of the linear model, that corresponds to the integration of (4), is given by

$$
\Delta v(t)=v_{0}+\delta v\left(1-\exp \left(-\frac{t-t_{s}}{\tau}\right)\right)
$$

where $\tau$ is the linear system time constant. The value of $\tau$ can be computed either graphically or analytically as the time instant when the velocity value is $\Delta v\left(t_{s}+\tau\right)=v_{0}+$ $\delta u\left(1-e^{-1}\right) \approx v_{0}+0.63 \delta u$.

Finally, parameter $a$ is the inverse of $\tau, a=\frac{1}{\tau}$, and $b$ is obtained from $K$ and $a$ as

$$
b=K a
$$

\section{Numerical algorithm for optimal control}

Consider the optimal control problem defined in appendix B. The numerical algorithm used to approximate the optimal control is a gradient based iterative method that proceeds until the stop criterion is met or the maximum number of iterations are reached. Each iteration consists of the following six sequential steps:

\section{Integrate state equation}

Using the current estimate of the optimal control signal, $u(t) \in \mathbb{R}^{m}$, integrate the state equation $\dot{x}=f(x, u, t)$ to obtain the state evolution $x(t) \in \mathbb{R}^{n}$ from $t_{0}$ to $t_{f}$.

\section{Integrate co-state equations}

Let $\lambda^{\Phi}(t)$, an $(n \times 1)$ vector, and $\lambda^{\Psi}(t)$, an $(n \times q)$ matrix, be co-state variables. Define the corresponding Hamiltonian functions as

$$
H^{\Phi}\left(\lambda^{\Phi}, x, u, t\right)=L(x, u, t)+\lambda^{\Phi^{T}} f(x, u, t),
$$




$$
H^{\Psi}\left(\lambda^{\Psi}, x, u, t\right)=\lambda^{\Psi^{T}} f(x, u, t),
$$

and integrate backwards, from $t_{f}$ to $t_{0}$, the co-state equations

$$
\begin{gathered}
-\left[\dot{\lambda}^{\Phi}\right]^{T}=H_{x}^{\Phi}=\frac{\partial L}{\partial x}+\lambda^{\Phi^{T}} \frac{\partial f}{\partial x} \\
-\left[\dot{\lambda}^{\Psi}\right]^{T}=H_{x}^{\Psi}=\lambda^{\Psi^{T}} \frac{\partial f}{\partial x}
\end{gathered}
$$

for which the terminal co-state conditions are

$$
\begin{aligned}
& \lambda^{\Phi}\left(t_{f}\right)=\phi_{x}^{T}\left(x\left(t_{f}\right), t_{f}\right), \\
& \lambda^{\Psi}\left(t_{f}\right)=\psi_{x}^{T}\left(x\left(t_{f}\right), t_{f}\right) .
\end{aligned}
$$

3. Compute Hamiltonian partial derivatives

Compute the Hamiltonian functions partial derivatives in respect to the control signal $u$ for all $t \in\left[t_{0}, t_{f}\right]$,

$$
\begin{gathered}
H_{u}^{\Phi}=\frac{\partial L}{\partial u}+\lambda^{\Phi^{T}} \frac{\partial f}{\partial u} \\
H_{u}^{\Psi}=\lambda^{\Psi^{T}} \frac{\partial f}{\partial u}
\end{gathered}
$$

where $H_{u}^{\Phi}(t)$ is a $(1 \times m)$ vector and $H_{u}^{\Psi}(t)$ is a $(q \times m)$ matrix.

\section{Compute Lagrange multiplier vector $\nu$}

Compute $\nu$ (a $q \times 1$ vector) by

$$
\nu=-Q^{-1} g
$$

where

$$
g=\int_{t_{0}}^{t_{f}} H_{u}^{\Psi}(t)\left[H_{u}^{\Phi}(t)\right]^{T} d t
$$

is a $(q \times 1)$ vector and

$$
Q=\int_{t_{0}}^{t_{f}} H_{u}^{\Psi}(t)\left[H_{u}^{\Psi}(t)\right]^{T} d t
$$

is a $(q \times q)$ matrix.

5. Compute control correction signal $\delta u(t)$

Evaluate $\psi$ at the terminal time and compute the control correction signal $\delta u(t)$ for all $t \in\left[t_{0}, t_{f}\right]$

$$
\delta u(t)=-k\left[H_{u}^{\Phi}(t)+\nu^{T} H_{u}^{\Psi}(t)\right]^{T}-\eta\left[H_{u}^{\Psi}(t)\right]^{T} Q^{-1} \psi\left(t_{f}\right)
$$

choosing $k<0(k>0)$ if maximizing (minimizing) the performance index, and $0<\eta \leq 1$.

Update the estimate of the optimal control signal

$$
u(t) \leftarrow u(t)+\delta u(t)
$$

\section{Evaluate stop criteria}

Compute the root-mean-square value of $\delta u(t)$

$$
\delta u_{r m s}=\frac{1}{t_{f}-t_{0}} \int_{t_{0}}^{t_{f}}[\delta u(t)]^{2} d t
$$

The algorithm stops if $\delta u_{r m s}$ is smaller than a specified threshold, or if the maximum number of iterations is reached.

\section{Minimum energy velocity transfer}

The minimum energy velocity transfer (MEVT) consists in transferring the vehicle velocity from a given initial value $v_{0}$ at $t=t_{0}$ to a desired final value $v_{f}$ at $t=t_{f}$, while minimizing a quadratic function fuel consumption.

This problem may not always be feasible. Depending on the maximum power available for the engine, there is a minimum value of the time interval required to transfer the velocity between two values. This interval depends on the starting velocity as well. Hereafter, we assume that the values specified for the transfer are such that this is feasible.

Here, the MEVT problem was considered with $t_{0}=0$ and $t_{f}=T$, for a given value of $T$, with starting velocity $v(0)=70 \mathrm{Km} / \mathrm{s}\left(u_{0} \approx 1.158 \times 10^{-3} \mathrm{~L} / \mathrm{s}\right)$ and a given final velocity $v(T)=v_{f}$. The linear model parameters used, $a=0.04167$ and $b=1774.97$.

For the linearized model, the performance index for solving the optimal MEVT problem is written as

$$
J_{l i n}(\Delta u)=-\frac{1}{2} \int_{0}^{T} \Delta u(t)^{2} d t
$$

in order to minimize the linearized model input: the incremental fuel flow $\Delta u(t)$. The maximization of this performance index yields the optimal incremental control signal $\Delta u_{l i n}^{*}(t)$. To obtain the optimal control signal $u_{l i n}^{*}$ we must add the working point fuel flow value $u_{0}$, i.e.

$$
u_{l i n}^{*}(t)=u_{0}+\Delta u_{l i n}^{*}(t)
$$

Thus, in order to allow the comparison between the optimal control signals for both models, nonlinear and linearized, the performance index for solving the optimal MEVT problem using the nonlinear model must also minimize the incremental control in respect to $u_{0}$, i.e.

$$
J(u)=-\frac{1}{2} \int_{0}^{T}\left(u(t)-u_{0}\right)^{2} d t .
$$

The maximization of this performance index yields the optimal control signal $u^{*}(t)$. This is because the linearized problem considers increments with respect to an equilibrium point, while the nonlinear formulation considers the full range of the variables.

Finally, both $u_{l i n}^{*}(t)$ and $u^{*}(t)$ are applied to the nonlinear car model, yielding state trajectories $v_{\text {lin }}^{*}$ and $v^{*}$, respectively. The corresponding cost functional value is also computed in both cases. Notice that using cost functional equation (11) with control signal $u_{\text {lin }}^{*}(t)$ is the same as using equation (9) with control signal $\Delta u_{\text {lin }}^{*}(t)$.

Results were obtained for some values of terminal time $T$, which are presented and discussed below.

\subsection{Velocity transfer with $T=300 \mathrm{~s}$}

With $T=300 \mathrm{~s}$, both systems have enough time to perform the velocity transfer from $v_{0}$ to $v_{f}$. In fact, careful inspection of the results shows that for the first half of the time in- 

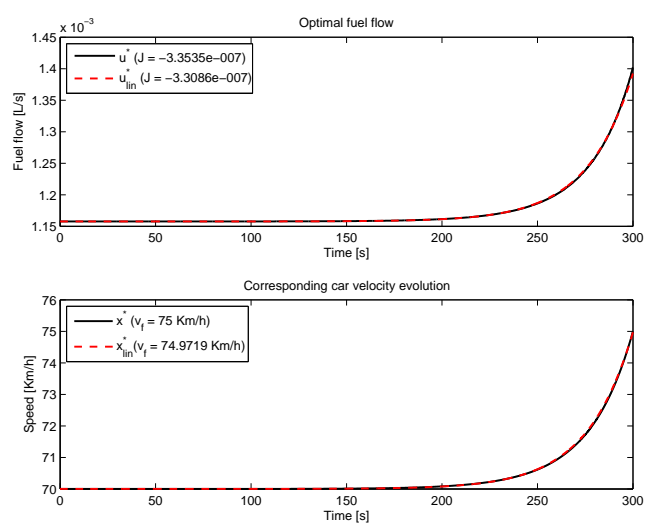

Figure 2. Velocity transfer result comparison for $T=300 \mathrm{~s}$ and $v_{f}=75 \mathrm{Km} / \mathrm{h}$.

terval under consideration the optimal control is fairly constant, equal to $u_{0} \approx 1.158 \times 10^{-3} \mathrm{~L} / \mathrm{s}$ in order to maintain the working point speed $v_{0}=70 \mathrm{Km} / \mathrm{h}$. Furthermore, for small values of $|\Delta v|=\left|v_{f}-v_{0}\right|$, the optimal control deviation from the working point fuel flow $u_{0}$, for which the linearization was designed, is lower and thus $u_{\text {lin }}^{*}(t) \approx u^{*}(t)$, naturally yielding $x_{l i n}^{*}(t) \approx x^{*}(t)$. This is the case in figure 2 . Figure 3 , where $v_{f}=90 \mathrm{Km} / \mathrm{h}$, shows that values of $v_{f}$ further away from $v_{0}$ lead to a higher deviation of the optimal control in respect to the working point $u_{0}$, and thus $u_{\text {lin }}^{*}(t)$ differs more significantly from $u^{*}(t)$. In other words, the nonlinear nature of the original model is more noticeable because we are further away from the linearization working point.

In the particular case of figure 3 , the performance index $J$ obtained for optimal control $u_{\text {lin }}^{*}(t)$ is actually higher than the one obtained for $u^{*}(t)$, but whereas the achieved final velocity of the latter is $v^{*}(300) \approx 90.00 \mathrm{Km} / \mathrm{h}$, the former meets the final velocity with much less accuracy, $v_{\text {lin }}^{*}(300) \approx 89.05 \mathrm{Km} / \mathrm{h}$, corresponding to an error . It is then clear that there exists a trade-off between computational effort, which is somewhat lighter when using the linearized model, and the precision in meeting the terminal state restrictions while minimizing fuel flow.

\subsection{Velocity transfer with $T=100 \mathrm{~s}$}

We have seen that for $T=300 \mathrm{~s}$ the optimal control signal needs only approximately 150 seconds to increase from $u_{0}$ to it's final value (slightly more for $v_{f}=90 \mathrm{~m} / \mathrm{s}$ ), to meet the terminal velocity constraint. By setting $T=100$ $\mathrm{s}$ the optimal control magnitude increases slightly, making it possible to meet the terminal velocity constraint in a smaller time interval.

When $v_{f}=75 \mathrm{Km} / \mathrm{h}$ (figure 4 ) the optimal control signals are, again, very similar. In fact, when using optimal control $u_{\text {lin }}^{*}$ the error obtained for the terminal velocity is only $0.0455 \%$. With $v_{f}=90 \mathrm{Km} / \mathrm{h}$ (figure 5) the non-
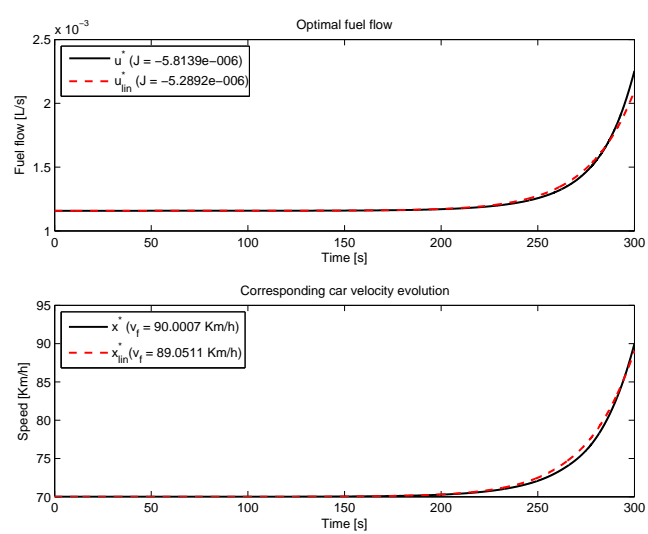

Figure 3. Velocity transfer result comparison for $T=300$ s and $v_{f}=90 \mathrm{Km} / \mathrm{h}$.
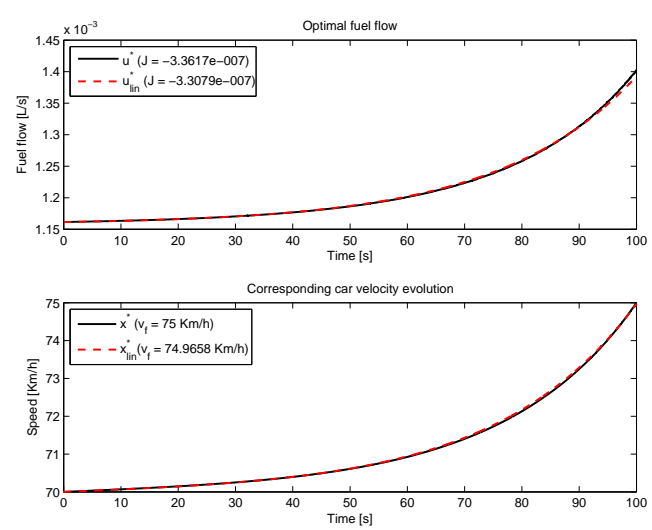

Figure 4. Velocity transfer result comparison for $T=100$ s and $v_{f}=75 \mathrm{Km} / \mathrm{h}$.

linear nature of the original model clearly shows and the resulting optimal control signals are again different. Like before, the performance index is slightly smaller for $u_{l i n}^{*}$ but the resulting final velocity is also smaller, with an error of $1.075 \%$.

\subsection{Velocity transfer with $T=10 \mathrm{~s}$}

The most noticeable result of reducing the terminal time to $T=10 \mathrm{~s}$ is the increase in the magnitude of the optimal control signal for both cases, $u^{*}$ and $u_{\text {lin }}^{*}$. For $v_{f}=75$ $\mathrm{Km} / \mathrm{h}$ (figure 6 ), both optimal control signals above $1.4 \times$ $10^{-3} \mathrm{~L} / \mathrm{s}$ whereas before (for $T=300,100 \mathrm{~s}$ ) they were below this value. The same happens when $v_{f}=90 \mathrm{Km} / \mathrm{h}$ (figure 7), for which $u^{*}$ and $u_{\text {lin }}^{*}$ are above $2.2 \times 10^{-3} \mathrm{~L} / \mathrm{s}$ whereas before they were mostly below this value.

As a result of the increase in the optimal fuel flow values, which are now further away from the working point fuel flow $u_{0}$ than in the previous cases, the discrepancy between $u^{*}$ and $u_{l i n}^{*}$ is clearly noticeable. In this situation, 

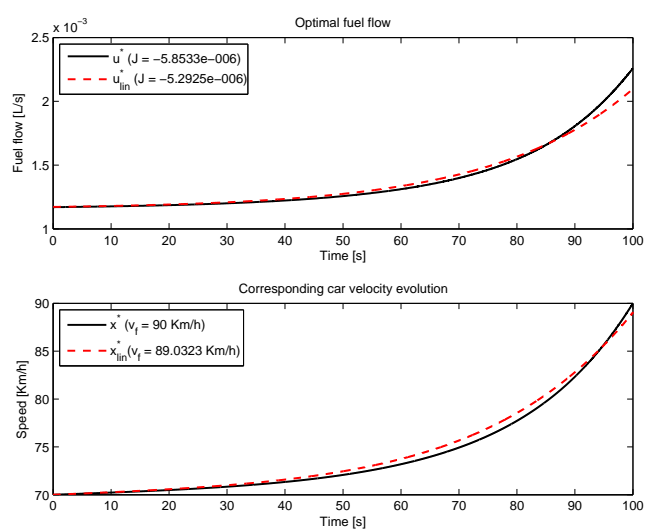

Figure 5. Velocity transfer result comparison for $T=100$ s and $v_{f}=90 \mathrm{Km} / \mathrm{h}$.
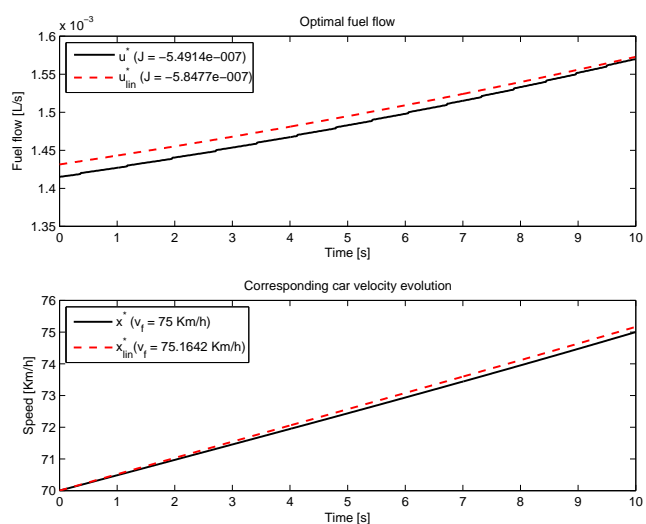

Figure 6. Velocity transfer result comparison for $T=10 \mathrm{~s}$ and $v_{f}=75 \mathrm{Km} / \mathrm{h}$.

the optimal control $u^{*}$ provides a better result, achieving the terminal velocity and a performance index that is higher than the one obtained by using the optimal control $u_{\text {lin }}^{*}$.

\section{Conclusions}

For situations that do not require the control signal to deviate much from the working point fuel flow $u_{0}$, the optimal control obtained from optimizing the linearized model, $u_{l i n}^{*}$, provides a reasonable, albeit less precise result. If precision is required, it is best to obtain the optimal control by optimizing the original nonlinear model, $u^{*}$.

Furthermore, since the fuel flow required to meet the terminal velocity increases when the terminal time $T$ is reduced beyond a certain threshold, special attention must be given to those situations. It is preferable to use optimal control $u^{*}$ in those cases.
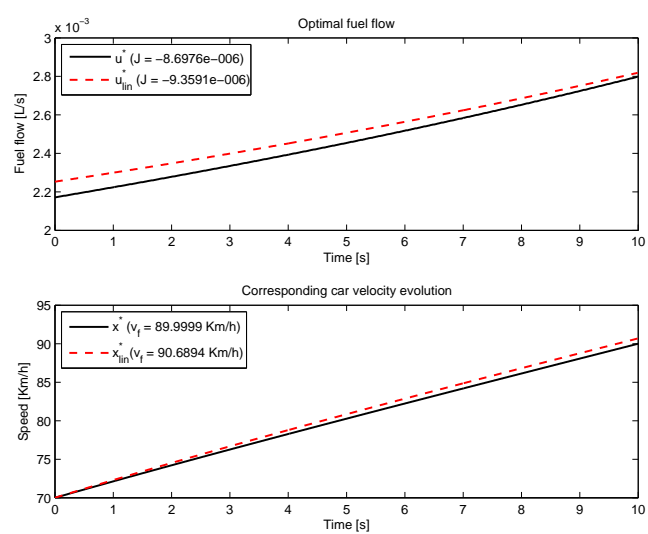

Figure 7. Velocity transfer result comparison for $T=10 \mathrm{~s}$ and $v_{f}=90 \mathrm{Km} / \mathrm{h}$.

\section{References}

[1] Ferreau, H. J., Lorini, G. and Diehl, M. (2006). Fast nonlinear model predictive control of gasoline engines. Proc. 2006 IEEE Int. Conf. on Control Applications, Munich, Germany, 2754-2759.

[2] Gausemeir, S., K.-P. Jaker and A. Trachtler (2010). Multi-objective Optimization of a Vehicle Velocity Profile by Means of Dynamic Programming. Prep. IFAC Symp. Advances in Automotive Control, AAC2010, Munich, Germany.

[3] Hashimoto, S. , Okuda, H., Okada, Y., Adachi, S., Niwa, S. and Kajitani, M. (2006). An engine control systems design for low emission vehicles by generalised predictive control based on identified model. Proc. 2006 IEEE Int. Conf. on Control Applications, Munich, Germany, 2411-2416.

[4] Kolmanovsky, I. V. and D. P. Filev (2010). Terrain and traffic Optimized Vehicle Speed Control. Prep. IFAC Symp. Advances in Automotive Control, AAC2010, Munich, Germany.

[5] Luu, H. T., L.Nouvelire and S. Mammar (2010). Dynamic Programming for fuel consumption optimization on light vehicle. Prep. IFAC Symp. Advances in Automotive Control, AAC2010, Munich, Germany.

[6] Saerens, B., J. Vandersteen, T. Persoons, M. Diehl and E. Van den Bulck (2009). Minimization of the fuel consumption of a gasoline engine using dynamic optimization. Applied Energy 86:15821588.

[7] T. Jorge, J. M. Lemos and M. Barão. Optimal cuise control with reduced complexity models. Prep. IFAC Symp. Advances in Automotive Control, AAC2010, Munich, Germany. 
[8] Bryson, A. E., and Ho, Y. C. (1975). Applied Optimal Control. Hemisphere, New York.

[9] Bryson, A. E. (1999). Dynamic Optimization. Addison-Wesley.

\section{A Nonlinear car model equations}

\subsection{Engine model}

The engine model assumes an input diesel flow $u(t)$ measured in liters per second. The total power is given by

$$
P(t)=E u(t)
$$

where $E$ is the total energy density of diesel fuel and $u$ is the diesel flow. A considerable percentage of this power is dissipated in thermal losses, and only a small part is available as mechanical power, which is thus given given by

$$
P_{m}(t)=\eta\left(T_{e}(t), w_{e}(t)\right) \times P(t) .
$$

The engine torque output $T_{e}$ is given by

$$
T_{e}(t)=\frac{P_{m}(t)}{\omega_{e}(t)}=\frac{\eta\left(T_{e}(t), w_{e}(t)\right) \times P(t)}{\omega_{e}(t)}
$$

Equation (14) constitutes an algebraic loop, since efficiency $\eta$ and engine torque values are computed based on each other, which makes computations more taxing. To overcome this, the torque value as a function of $w_{e}(t)$ and $u(t)$ can be numerically computed, by solving the algebraic loop (14) offline.

For the purposes of this work, it was assumed that efficiency level-curves on the $\left(T_{e}, w_{e}\right)$ plane are given by

$$
\eta\left(T_{e}, w_{e}\right)=\alpha-\beta\left[\frac{\left(T_{e}-c_{T}\right)^{2}}{l_{T}}+\frac{\left(w_{e}-c_{w}\right)^{2}}{l_{w}}\right]
$$

for a reasonable choice of $c_{T}, l_{T}, c_{w}$ and $l_{w}$. Constants $\alpha$ and $\beta$ perform a linear transformation, making the elliptic surface concavity face downwards instead of upwards. Constant $\alpha$ is the value of the maximum efficiency, i.e. when $\left(T_{e}, w_{e}\right)=\left(c_{T}, c_{w}\right)$ then $\eta=\alpha$.

In this specific case a closed-form solution for computing $T_{e}(t)$ can be easily derived by replacing (15) in (14). While this is not the general case, it is important to notice that a numerical solution with sufficient precision is enough.

From the data available for this engine, it is known that it achieves a maximum torque of $310 \mathrm{Nm}$ at $1800-2400$ rpm. Below and above this operational range the torque is greatly reduced. It is also known that a maximum power of $93 \mathrm{~kW}$ is attained at $3600 \mathrm{rpm}$, implying a torque $T=$ $\frac{93 \times 10^{3}}{3600} \frac{60}{2 \pi} \approx 246.7 \mathrm{Nm}$ at that speed.

From this scarce data, a maximum torque curve was designed. For any given engine speed, admissible engine torque values lie below this curve.

\subsection{Transmission}

The transmission links the wheels and the engine together using a gear box. Its role is to increase torque and decrease wheel speed to match the operational range of the engine. The transmission also introduces internal drag that depends on the engine speed. In the model developed here, the internal drag does not only model the transmission itself, but also all the load at the engine shaft.

The torque output available at the car wheels is given by

$$
T_{w}(t)=r_{i} r_{f}\left(T_{e}(t)-\alpha-\beta \omega_{e}(t)\right)
$$

where $r_{i}$ is the gear ratio for gear $i, r_{f}$ is the final drive ratio, $\alpha, \beta$ are drag coefficients and $T_{e}(t), \omega_{e}(t)$ are the engine torque and speed, respectively.

Engine rotational speed, measured in $[\mathrm{rad} / \mathrm{s}]$, is obtained from wheel speed by gear ratio conversion and is given by

$$
\omega_{e}(t)=r_{i} r_{f} \omega_{w}(t)
$$

\subsection{Traction force and wheels}

The wheels are modeled as a rotational to linear movement converter neglecting inertia and drag. Wheel rotational speed, measured in $[\mathrm{rad} / \mathrm{s}]$, is given by

$$
\omega_{w}(t)=\frac{2 \pi}{P} v(t)
$$

where $\mathrm{P}$ is the wheel perimeter. The used tire dimensions are 205/55R 16 , corresponding to a perimeter of $P=$ 1.9852 meters.

Similarly, traction force is obtained from the torque applied by the engine at the wheels

$$
F(t)=\frac{2 \pi}{P} T_{w}(t)
$$

\section{B Iterative numeric solution of the optimal control problem with terminal constraints}

Using the methods of $[8,9]$, this appendix shows how to construct a history $\delta u(t)$ that optimizes the performance index by making the control signal converge to the optimal control $u^{*}(t)$. To simplify the notation, we shall make $\frac{\partial f}{\partial x} \equiv f_{x}, \frac{\partial f}{\partial u} \equiv f_{u}, \frac{\partial L}{\partial x} \equiv L_{x}$ and $\frac{\partial L}{\partial u} \equiv L_{u}$.

Let

$$
\dot{x}=f(x, u, t), \quad t \in\left[t_{0}, t_{f}\right]
$$

describe the generally nonlinear time-varying dynamics of a plant, where $x(t) \in \mathbb{R}^{n}$ and $u(t) \in \mathbb{R}^{m}$ are the statevector and input vector at time $t$, respectively, and

$$
J=\phi\left(x\left(t_{f}\right), t_{f}\right)+\int_{t_{0}}^{t_{f}} L(x(t), u(t), t) d t
$$

a performance index, associated with the above plant, which we wish to maximize. A minimization problem 
can also be formulated by maximizing the performance index $J_{\min }=-J$. Notice that function $\phi\left(x\left(t_{f}\right), t_{f}\right)$ is a function of the terminal state and terminal time, while $L(x(t), u(t), t)$ depends on the state, input and time values in the interval $\left[t_{0}, t_{f}\right]$.

The optimal control problem consists in finding input signal $u^{*}(t), t \in\left[t_{0}, t_{f}\right]$, for which the plant exhibits a state trajectory $x^{*}(t)$, such that the cost functional value, $J$, is maximum. Additionally, one might wish to introduce restrictions on the terminal state value, in the form of $q$ restriction equations

$$
\psi\left(x\left(t_{f}\right), t_{f}\right)=\left[\begin{array}{c}
\psi_{1}\left(x\left(t_{f}\right), t_{f}\right) \\
\cdots \\
\psi_{q}\left(x\left(t_{f}\right), t_{f}\right)
\end{array}\right]=\left[\begin{array}{c}
0 \\
\cdots \\
0
\end{array}\right] .
$$

The problem is thus to maximize the performance index

(21) subject to constraints (20) and (22).

As is well known, the solution to this problem satisfies the set of necessary conditions:

1. State equation

$H_{\lambda}-\dot{x}^{T}=0 \Leftrightarrow \dot{x}=H_{\lambda}^{T}=f(x(t), u(t), t)$,

\section{Co-state equation}

$H_{x}+\dot{\lambda}^{T}=0 \Leftrightarrow-\dot{\lambda}^{T}=H_{x}=\frac{\partial L}{\partial x}+\lambda^{T} \frac{\partial f}{\partial x}$,

3. Final state condition

$$
\psi\left(x\left(t_{f}\right), t_{f}\right)=0
$$

\section{Stationarity condition}

$$
H_{u}=0 \Leftrightarrow \frac{\partial H}{\partial u}=\frac{\partial L}{\partial u}+\lambda^{T} \frac{\partial f}{\partial u},
$$

\section{Boundary condition}

$$
\left[\left(\phi_{x}+\nu^{T} \psi_{x}-\lambda^{T}\right) d x\right]_{t=t_{f}}=0,
$$

Assume now that we wish to constrain the $i$ th component of the state vector by prescribing a fixed value at the terminal time, $x_{i}\left(t_{f}\right)$. It follows that $d x_{i}\left(t_{f}\right)=0$ and thus in order to satisfy the boundary equation (27) it is not necessary that $\left[\frac{\partial \phi}{\partial x_{i}}-\lambda_{i}^{T}\right]_{t=t_{f}}=0$. In fact, we have simply traded this latter boundary condition for another one, namely $x_{i}\left(t_{f}\right)$ given. If we wish to prescribe a fixed value at the terminal time for first $q$ components of the state vector, then

$$
\delta x_{i}\left(t_{f}\right)=0, \quad i=1, \ldots, q
$$

and thus $\phi$ is a function of the remaining components of the state vector

$$
\phi=\phi\left(x_{q+1}, \ldots, x_{n}\right)_{t=t_{f}} .
$$

It is more general to prescribe a fixed value for the terminal state by means of a function written in the format

$$
\psi_{i}\left(x_{i}\left(t_{f}\right), t_{f}\right)=0, \quad i=1, \ldots, q \text {. }
$$

for which, if the terminal time $t_{f}$ is fixed, we can derive the following relation

$$
\delta \psi_{i}\left(x_{i}\left(t_{f}\right), t_{f}\right)=0 \Leftrightarrow \delta x_{i}\left(t_{f}\right)=0
$$

Henceforth we shall use $\psi_{i}\left(t_{f}\right) \equiv \psi_{i}\left(x_{i}\left(t_{f}\right), t_{f}\right)$ to simplify notation. We derive now an equation for the variation of the augmented performance

$$
\begin{gathered}
d \bar{J}=\left[\left(\phi_{x}-\lambda^{\Phi^{T}}\right) d x\right]_{t=t_{f}}+ \\
+\int_{t_{0}}^{t_{f}}\left[\left(H_{x}^{\Phi}+\left[\dot{\lambda}^{\Phi}\right]^{T}\right) \delta x+H_{u}^{\Phi} \delta u+\left(H_{\lambda^{\Phi}}^{\Phi}-\dot{x}^{T}\right) \delta \lambda^{\Phi}\right] d t
\end{gathered}
$$

where the superscript $\Phi$ is used to differentiate from analogous variables introduced later.

Knowing that the first $q$ components of the state vector are prescribed at the terminal time

$$
\psi\left(x\left(t_{f}\right), t_{f}\right)=\left[\begin{array}{c}
\psi_{1}\left(t_{f}\right) \\
\psi_{2}\left(t_{f}\right) \\
\vdots \\
\psi_{q}\left(t_{f}\right)
\end{array}\right]=\left[\begin{array}{c}
0 \\
0 \\
\vdots \\
0
\end{array}\right],
$$

by replacing equations (23), (24) and (27) into (32) it follows that

$$
\begin{gathered}
d \bar{J}=\int_{t_{0}}^{t_{f}}\left[H_{u}^{\Phi} \delta u\right] d t= \\
\int_{t_{0}}^{t_{f}}\left[\left(L_{u}+\lambda^{\Phi^{T}} f_{u}\right) \delta u\right] d t
\end{gathered}
$$

where

$$
\begin{gathered}
H^{\Phi}=L+\lambda^{\Phi^{T}} f \\
-\left[\dot{\lambda}^{\Phi}\right]^{T}=H_{x}^{\Phi}=L_{x}+\lambda^{\Phi^{T}} f_{x},
\end{gathered}
$$

$$
\lambda_{i}^{\Phi}\left(t_{f}\right)=\left\{\begin{array}{clrl}
0, & & i=1, \ldots, q \\
\left(\frac{\partial \phi}{\partial x_{i}}\right)_{t_{f}}, & i & =q+1, \ldots, n
\end{array}\right.
$$

Assume that, instead of (21), the performance index was $J^{\prime}=\psi_{i}\left(x_{i}\left(t_{f}\right), t_{f}\right)$, i.e. the function that prescribes the $i$ th component of the state vector at the terminal time. This corresponds to making the objective function $\phi^{\prime}\left(x\left(t_{f}\right), t_{f}\right)=\psi_{i}\left(t_{f}\right)$ and the lagrangian function $L^{\prime}(x, u, t)=0$. Then the equivalent expressions to (34), (36), and (37) can be written as

$$
\begin{gathered}
d \bar{J}^{\prime}=\delta \psi_{i}\left(t_{f}\right)=\int_{t_{0}}^{t_{f}}\left[H_{u}^{(i)} \delta u\right] d t= \\
\int_{t_{0}}^{t_{f}}\left[\left(\lambda^{(i)^{T}} f_{u}\right) \delta u\right] d t
\end{gathered}
$$

where

$$
\begin{gathered}
H^{(i)}=\lambda^{(i)^{T}} f, \\
-\left[\dot{\lambda}^{(i)}\right]^{T}=H_{x}^{(i)}=\lambda^{(i)^{T}} f_{x},
\end{gathered}
$$




$$
\lambda_{k}^{(i)}\left(t_{f}\right)=\left\{\begin{array}{ll}
0, & k \neq i \\
\left(\frac{\partial \psi_{i}}{\partial x_{i}}\right)_{t_{f}}, & k=i
\end{array} \quad k=1, \ldots, n\right.
$$

We shall now construct a history $\delta u(t)$ that increases $\mathbf{J}$ $(d \bar{J}>0)$ and satisfies the $q$ terminal constraints (30). Multiply each of the $q$ equations (38) by an undetermined constant $\nu_{i}$, and add the resulting equations to (34)

$$
\begin{gathered}
d \bar{J}+\sum_{i=1}^{q}\left\{\nu_{i} \delta \psi_{i}\left(t_{f}\right)\right\}=d \bar{J}+\nu^{T} \delta \psi\left(t_{f}\right) \\
\int_{t_{0}}^{t_{f}}\left(L_{u}+\left[\lambda^{\Phi}+\lambda^{q}\right]^{T} f_{u}\right) \delta u d t
\end{gathered}
$$

where

$$
\begin{gathered}
\nu=\left[\begin{array}{cccc}
\nu_{1} & \nu_{2} & \cdots & \nu_{q}
\end{array}\right]^{T}, \\
\lambda^{q}=\sum_{i=1}^{q}\left\{\nu_{i} \lambda^{(i)}\right\},
\end{gathered}
$$

and $\psi\left(t_{f}\right) \equiv \psi\left(x\left(t_{f}\right), t_{f}\right)$ to simplify notation. Now, choose

$$
\delta_{u}=-k\left(f_{u}^{T}\left[\lambda^{\Phi}+\lambda^{q}\right]+L_{u}^{T}\right)
$$

where $k$ is a negative scalar constant, and substitute this expression into (42), as follows

$$
\begin{gathered}
d \bar{J}+\nu^{T} \delta \psi\left(t_{f}\right)= \\
-k \int_{t_{0}}^{t_{f}}\left\|f_{u}^{T}\left[\lambda^{\Phi}+\lambda^{q}\right]+L_{u}^{T}\right\|^{2} d t
\end{gathered}
$$

that is always positive unless the integrand vanishes over the whole integration interval. Next we determine the value of constants $\nu_{i}$ in order to satisfy the terminal constraints (30). Substituting (45) into (38), we have

$$
\begin{gathered}
0=\delta \psi_{i}\left(t_{f}\right) \Leftrightarrow \\
\Leftrightarrow 0=-k \int_{t_{0}}^{t_{f}} \lambda^{(i)^{T}} f_{u}\left(f_{u}^{T}\left[\lambda^{\Phi}+\lambda^{q}\right]+L_{u}^{T}\right) d t \\
\Leftrightarrow 0=\int_{t_{0}}^{t_{f}} \lambda^{(i)^{T}} f_{u}\left[f_{u}^{T} \lambda^{\Phi}+L_{u}^{T}\right] d t+\int_{t_{0}}^{t_{f}} \lambda^{(i)^{T}} f_{u} f_{u}^{T} \lambda^{q} d t \\
\Leftrightarrow 0=\int_{t_{0}}^{t_{f}} \lambda^{(i)^{T}} f_{u}\left[f_{u}^{T} \lambda^{\Phi}+L_{u}^{T}\right] d t+ \\
+\sum_{j=1}^{q}\left\{\nu_{j} \int_{t_{0}}^{t_{f}} \lambda^{(i)^{T}} f_{u} f_{u}^{T} \lambda^{(j)} d t\right\} \\
\Leftrightarrow 0=g_{i}+\sum_{j=1}^{q}\left\{\nu_{j} Q_{i j}\right\}
\end{gathered}
$$

where

$$
g_{i}=\int_{t_{0}}^{t_{f}} \lambda^{(i)^{T}} f_{u}\left[f_{u}^{T} \lambda^{\Phi}+L_{u}^{T}\right] d t=
$$

$$
\begin{gathered}
\int_{t_{0}}^{t_{f}} H_{u}^{(i)}\left[H_{u}^{\Phi}\right]^{T} d t \\
Q_{i j}=\int_{t_{0}}^{t_{f}} \lambda^{(i)^{T}} f_{u} f_{u}^{T} \lambda^{(j)} d t= \\
\int_{t_{0}}^{t_{f}} H_{u}^{(i)}\left[H_{u}^{(j)}\right]^{T} d t .
\end{gathered}
$$

The $q$ equations (47) can be written in matrix format as

$$
0=g+Q \nu
$$

where $Q$ is the $(q \times q)$ terminal state controllability matrix. By defining

$$
\lambda^{\Psi}=\left[\begin{array}{llll}
\lambda^{(1)} & \lambda^{(2)} & \cdots & \lambda^{(q)}
\end{array}\right]
$$

an $(n \times q)$ matrix and

$$
H^{\Psi}=\left[\begin{array}{llll}
H^{(1)} & H^{(2)} & \cdots & H^{(q)}
\end{array}\right]^{T}=\lambda^{\Psi^{T}} f
$$

a $q$-component column array, one can also write

$$
\begin{gathered}
g=\int_{t_{0}}^{t_{f}} H_{u}^{\Psi}\left[H_{u}^{\Phi}\right]^{T} d t, \\
Q=\int_{t_{0}}^{t_{f}} H_{u}^{\Psi}\left[H_{u}^{\Psi}\right]^{T} d t .
\end{gathered}
$$

The appropriate choice for the multipliers $\nu_{j}$ is then

$$
\nu=-Q^{-1} g
$$

If $Q$ is a singular matrix, $Q^{-1}$ does not exist, meaning that it is not possible to control the system with $u(t)$ in order to satisfy one or more of the terminal conditions.

We have thus constructed a $\delta u(t)$ history that increases the performance index and satisfies the terminal constraints (30). From (46) the only case in which we cannot increase the performance index is when

$$
L_{u}+\left[\lambda^{\Phi}+\lambda^{q}\right]^{T} f_{u}=0, \quad t_{0} \leq t \leq t_{f}
$$

If (55) is satisfied, we have a stationary solution that satisfies the terminal constraints. 